\title{
Proteomic analysis of human synovial fluid reveals potential diagnostic biomarkers for ankylosing spondylitis
}

Ji-Hyun Lee ${ }^{1 \dagger}$, Jae Hun Jung ${ }^{2 \dagger}$, Jeesoo Kim³ ${ }^{3,4}$, Won-Ki Baek ${ }^{5}$, Jinseol Rhee ${ }^{6}$, Tae-Hwan Kim, Sang-Hyon Kim', Kwang Pyo Kim ${ }^{2 *}$ (D), Chang-Nam Son ${ }^{1^{*}}$ (D) and Jong-Seo Kim ${ }^{3,4^{*}}$ (D)

\begin{abstract}
Background: Ankylosing spondylitis (AS) is a chronic inflammatory rheumatic disease affecting the axial skeleton and peripheral joints. The etiology of this disease remains poorly understood, but interactions between genetic and environmental factors have been implicated. The present study identified differentially expressed proteins in the synovial fluid (SF) of AS patients to elucidate the underlying cause of AS.
\end{abstract}

Methods: A cohort of $40 \mathrm{SF}$ samples from $10 \mathrm{AS}$ and 10 each of rheumatoid arthritis (RA), gout, and osteoarthritis (OA) patients were analyzed by liquid chromatography tandem mass spectrometry (LC-MS/MS) to identify differentially expressed proteins specific to AS. The label-free LC-MS/MS results were verified by western blotting.

Results: We identified 8 proteins that were $>1.5$-fold upregulated in the SF of AS patients compared to that of the disease control groups, including HP, MMP1, MMP3, serum amyloid P-component (APCS), complement factor H-related protein 5 (CFHR5), mannose-binding lectin 2 (MBL2), complement component C9 (C9), and complement C4-A (C4A). CFHR5 and C9 were previously found in serum from AS patients, while APCS was previously found in SF as well as in serum. However, the present study has identified C4A, and MBL2 as potential AS biomarkers for the first time. The expression levels of MMP3, C9, and CFHR5 were verified in AS SF using western blotting.

Conclusion: We performed quantitative comparative proteomic analysis using by LC-MS/MS of the SF from four disease states: RA, gout, and OA. This systematic comparison revealed novel differentially expressed proteins in AS SF, as well as two previously reported candidate biomarkers. We further verified the expression of MMP3, C9 and CFHR5 by western blot. These proteins may serve as diagnostic or prognostic biomarkers in patients with AS, and may thus improve the clinical outcomes of this serious disease.

Keywords: Ankylosing spondylitis, Proteomics, Synovial fluid, Biomarker

*Correspondence: kimkp@khu.ac.kr; cnson@kmu.ac.kr; jongseokim@snu.ac.kr ${ }^{\dagger} \mathrm{Ji}$-Hyun Lee and Jae Hun Jung equally contributed to this work

${ }^{1}$ Division of Rheumatology, Department of Internal Medicine, School

of Medicine, Keimyung University, Daegu, South Korea

${ }^{2}$ Department of Applied Chemistry, Institute of Natural Science, Global

Center for Pharmaceutical Ingredient Materials, Kyung Hee University, Yongin, South Korea

${ }^{4}$ School of Biological Sciences, Seoul National University, Seoul 08826, South Korea

Full list of author information is available at the end of the article

\section{Background}

Ankylosing spondylitis (AS) is a chronic inflammatory rheumatic disease, affecting the axial skeleton and peripheral joints, that occurs in $0.5 \%$ of the general population [1]. Without proper clinical treatment, AS causes permanent structural changes, leading to progressive disability that affects the quality of life [2]. While the exact etiology of this disease remains poorly understood, it may be caused by interactions between genetic and

(c) The Author(s) 2020. This article is licensed under a Creative Commons Attribution 4.0 International License, which permits use, sharing, adaptation, distribution and reproduction in any medium or format, as long as you give appropriate credit to the original author(s) and the source, provide a link to the Creative Commons licence, and indicate if changes were made. The images or other third party material in this article are included in the article's Creative Commons licence, unless indicated otherwise in a credit line to the material. If material is not included in the article's Creative Commons licence and your intended use is not permitted by statutory regulation or exceeds the permitted use, you will need to obtain permission directly from the copyright holder. To view a copy of this licence, visit http://creativeco mmons.org/licenses/by/4.0/. The Creative Commons Public Domain Dedication waiver (http://creativecommons.org/publicdomain/ zero/1.0/) applies to the data made available in this article, unless otherwise stated in a credit line to the data. 
environmental factors [3]. AS represents an increasing financial burden on both patients and the healthcare system $[4,5]$.

AS is defined by the presence of definite structural changes in the sacroiliac joints. Although several possibilities for the etiology of AS, including human leukocyte antigen (HLA)-B27 and tumor necrosis factor-alpha (TNF- $\alpha$ ), have been proposed as a therapeutic target $[2$, $4,5]$, no clear cause for AS has been identified. Peripheral arthritis is an important feature of spondyloarthritis $(\mathrm{SpA})$ in general $[5,6]$. Swelling of the knees with inflammatory pain is a representative symptom of peripheral arthritis, and work-up for peripheral arthritis can identify all forms of SpA early and differentiate it from other arthritides. The synovial fluid (SF) from the knees may therefore contain diagnostic biomarkers for AS disease.

SF lubricates the joints, and consists of hyaluronic acid, inflammatory cells, and secreted proteins from the synovial fibroblasts, synovial membranes, and inflammatory cells [7]. Normally, SF is present in small amounts in all joints, acting as joint lubricant and providing nutrition for articular cartilage. However, when inflammation occurs, synovial cells secrete a large amount of synovial fluid and become the subjects of an inflammatory reaction that destroys the joints. Arthrocentesis is the extraction of synovial fluid and has been used for differential diagnosis in patients with inflammatory arthritis [8]. Although serologic and imaging techniques have been developed to diagnose rheumatic diseases, synovial fluid analysis is still used as an important diagnostic tool for differential diagnosis of arthritis, especially acute arthritic disease, since SF is in contact with the primary tissues affected by arthritic diseases and is implicated in the disease pathophysiology. Therefore, it is an excellent biofluid for the discovery of candidate biomarkers in arthritis-related diseases like AS [9].

In the last decade, many proteomic studies in the area of rheumatic diseases have been published [10-14], largely with the aim of diagnosing disease and evaluating disease activity/severity and therapeutic response $[11,12]$. Different rheumatic disease samples with complicated pathologic structure, such as blood, SF, synovial tissue, and urine, have been investigated for system-wide discovery and validation of rheumatoid arthritis biomarkers [15]. Currently used diagnostic tests for rheumatoid arthritis (RA) include rheumatoid factor and anti-CCP antibodies (anti-cyclic citrullinated peptide antibodies, anti-CCP Ab) [16]. However, there is still no clinically available protein biomarker for early diagnosis and monitoring of AS. Although HLAB27 is currently used for AS diagnosis, it has a high incidence of false positives. Although there were differences by race, the frequency of HLA-B27 was $4 \%$ in the normal control group and $83.3 \%$ in the patients with ankylosing spondylitis. Patients with negative HLA-B27 tend to be delayed in diagnosis because the symptoms are not typical. Therefore, the discovery of new robust biomarkers for AS is required for effective early diagnosis and treatment. In the present study, we performed a quantitative proteomics comparison of SF proteins isolated from AS patients [17] and three other arthritis patient groups (RA, gout, and OA). OA is a non-inflammatory arthritis, and was included as a disease control for inflammatory arthritic diseases [18], while both RA and gout were disease controls for inflammatory arthritis from different pathologic origins [19]. Following immunodepletion of super-abundant proteins and label-free quantitative proteomics, we found eight ASspecific and differentially expressed proteins in the AS group compared to the disease control groups. Further western blot verification confirmed the discriminatory ability of seven of these proteins in AS patients. Among these, MMP3 (Matrix metalloproteinase-3), C9 (complement component C9) and CFHR5 (Complement factor H-related protein 5) are upregulated in the SF of AS patients, and could therefore be potential biomarkers for AS diagnosis.

\section{Methods \\ Subjects}

Ten patients with AS were recruited from an outpatient rheumatology clinic in Hanyang University Hospital for Rheumatic Diseases, Seoul, South Korea. An additional 30 patients treated at Keimyung University Dongsan Hospital, Daegu, South Korea were recruited as disease control groups (10 OA, 10 gout, and $10 \mathrm{RA})$. These patient SF samples (first cohort) were used for the LCMS/MS experiments and the associated western blot verification. We further recruited five more patient samples per each patient group (second cohort) for the independent western blot verification experiments (5 AS, 5 OA, 5 gout, and 5 RA). The patients met the 1984 New York criteria for ankylosing spondylitis [17] and the American College of Rheumatology criteria for RA [16], knee OA [18], and acute gout [19]. We collected demographic and clinical data from the subjects, including age, gender, disease duration, blood chemistry, and concomitant treatment. We provide the summary statistics (Table 1) and the original data including the diagnosis, clinical data, and current treatments of each patient enrolled in this study (Additional file 1: Table S1). The samples were obtained after getting informed consent from the patients. The study was approved by the ethical committee of the Keimyung University Dongsan Hospital (IRB 2015-12-022). 
Table 1 The clinical and laboratory features of patients with ankylosing spondylitis, rheumatoid arthritis, gout, and osteoarthritis

\begin{tabular}{lllll}
\hline Parameters & Ankylosing spondylitis & Rheumatoid arthritis & Gout & Osteoarthritis \\
\hline No. of patients & 10 & 10 & 10 & 10 \\
Age (years) & $32.6 \pm 10.6$ & $59.6 \pm 11.6$ & $62.8 \pm 12.8$ & $64.8 \pm 8.5$ \\
Sex (M/F) & $9 / 1$ & $0 / 10$ & $10 / 0$ & $1 / 9$ \\
ESR (mm/h) & $59.5 \pm 39.7$ & $32.7 \pm 17.0$ & $69 \pm 21.3(\mathrm{n}=5)$ & $27.5 \pm 15.2(\mathrm{n}=4)$ \\
CRP $(\mathrm{mg} / \mathrm{dL})$ & $4.9 \pm 4.3$ & $0.5 \pm 3.7$ & $3.4 \pm 3.7(\mathrm{n}=6)$ & $0.1 \pm 0.04(\mathrm{n}=4)$ \\
\hline
\end{tabular}

CRP: C-reactive protein; ESR: erythrocyte sedimentation rate; F: female; M: male

\section{Synovial fluid sample collection and processing}

Synovial fluid was collected by arthrocentesis in patients with knee joint pain and swelling. Contaminated bloods during arthrocentesis and samples where synovial fluids were generated for reasons other than the respective diseases were excluded. After centrifugation at $500 \times g$ for $10 \mathrm{~min}$, five $1 \mathrm{~mL}$ vials of supernatant and 1 vial of sediment from each sample were stored at $-80{ }^{\circ} \mathrm{C}$ through the Keimyung University Dongsan Hospital Human Resource Bank.

\section{Immunodepletion of abundant proteins with MARS cartridge}

We used a Multiple Affinity Removal System (MARS) $\mathrm{Hu}-14$ spin cartridge (Agilent, 5188-6560), which contains a bulk of immobilized antibodies against the 14 most abundant proteins (albumin, IgG, antitrypsin, IgA, transferrin, haptoglobin, fibrinogen, alpha-2-macroglobulin, alpha-1-acid glycoprotein, IgM, apolipoprotein A1, apolipoprotein A2, complement C3 and transthyretin), which are known to occupy about $95 \%$ of plasma proteome [20], enabling immunodepletion of such abundant proteins in SF. The $0.22 \mu \mathrm{m}$ membrane filter (Agilent) was used to remove particulates from the fluid samples by centrifugation at $100 \mathrm{xg}$ for $1.5 \mathrm{~min}$. The flow-through was mixed with Buffer A LOAD/WASH (Agilent) and depleted according to the manufacturer's instructions. During the depletion, flow-through was collected, and protein concentration was determined using a BCA assay. The column was routinely regenerated by eluting bound high-abundance proteins with buffer B and neutralizing with buffer A before further use. The acquired proteins were directly digested for total proteomic analysis.

\section{Peptide sample preparation}

In-solution tryptic digestion and peptide cleanup were simultaneously performed in a 96-well plate for high reproducibility. Each depleted sample was supplemented with $8 \mathrm{M}$ urea in $100 \mathrm{mM}$ ammonium bicarbonate (ABC) (Sigma) and incubated at room temperature for $20 \mathrm{~min}$.
The samples were homogenized by vortexing and sonication twice. To each sample, dithiothreitol (Sigma) was added to be $10 \mathrm{mM}$ for protein reduction at RT for $1 \mathrm{~h}$. Then, iodoacetamide (Sigma) was added to be $30 \mathrm{mM}$ for the cysteine alkylation at RT for $30 \mathrm{~min}$ in the dark. Samples were then diluted with $100 \mathrm{mM} \mathrm{ABC}$ prior to the addition of trypsin (MS grade, Pierce) at 1:50 of trypsin:sample ratio $(\mathrm{w} / \mathrm{w})$, and incubated at $37{ }^{\circ} \mathrm{C}$ for overnight. The trypsin was inactivated by acidification with $0.4 \%$ trifluoroacetic acid (Sigma). The acidified digests were immediately processed using a Sep-Pak C18 96-well plate (100 mg C18 sorbent per well, Waters). The peptides were eluted with $80 \%$ acetonitrile and then dried in a vacuum centrifuge.

\section{LC-MS/MS experiments}

We performed a label-free quantitative proteomics using LC-MS/MS experiments in synovial fluid samples. Forty LC-MS/MS experiments were carried out on an Orbitrap Fusion Lumos mass spectrometer (Thermo Fisher Scientific) coupled to a nanoACQUITY UPLC (Waters) with an in-house-packed trap $(150 \mu \mathrm{m}$ i.d. $\times 3 \mathrm{~cm})$ and analytical column $(75 \mu \mathrm{m}$ i.d. $\times 100 \mathrm{~cm})$ using $3 \mu \mathrm{m}$ Jupiter $\mathrm{C} 18$ particles (Phenomenex). The LC gradient was as follows: from $5 \%$ to $40 \%$ solvent B (acetonitrile with $0.1 \%$ formic acid) for $130 \mathrm{~min}$, then $40 \%$ to $80 \%$ solvent B for 5 min, holding at $80 \%$ solvent B for $10 \mathrm{~min}$, and then equilibrating at $95 \%$ solvent $A$ (water with $0.1 \%$ formic acid) for $30 \mathrm{~min}$. Full MS data were acquired in a scan range of $375-1575 \mathrm{Th}$ at a resolution of 60,000 at $\mathrm{m} / \mathrm{z}$ 200 , with an automated gain control (AGC) target value of $4.0 \times 10^{5}$ and a maximum ion injection time of $50 \mathrm{~ms}$. The maximal ion injection time for MS/MS was $50 \mathrm{~ms}$ at a resolution of 15,000 and an AGC target value of 5 $\times 10^{4}$. The dynamic exclusion time was set to $30 \mathrm{~s}$. The resulting forty MS raw files were analyzed using MaxLFQ in Maxquant software (Fig. 1).

\section{Data analysis}

MaxQuant (v.1.5.1.2) [21] was used to compare the acquired spectra to the UniProt human database 


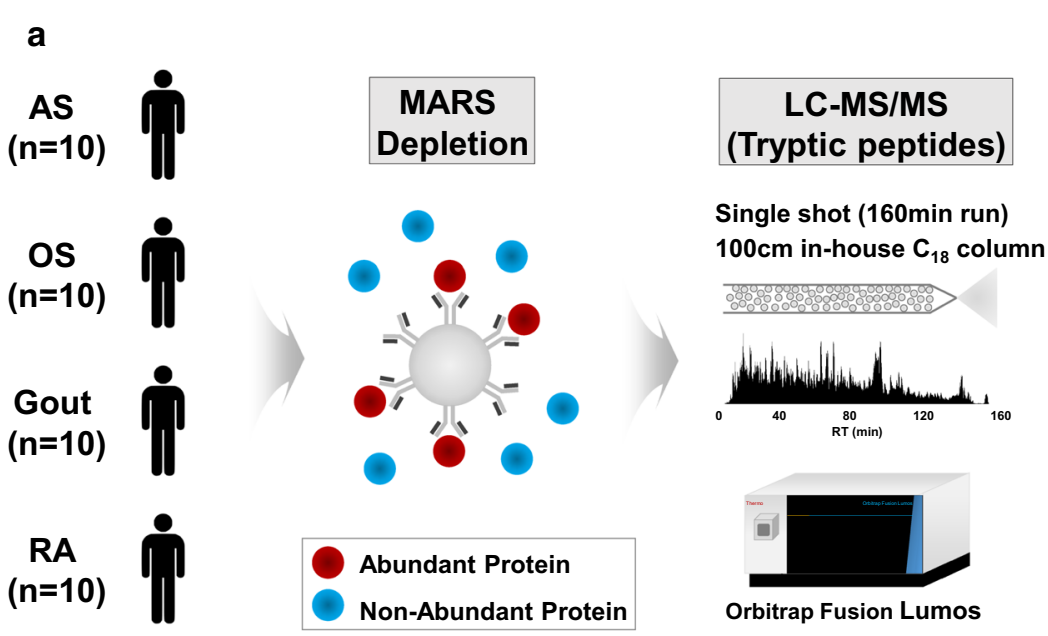

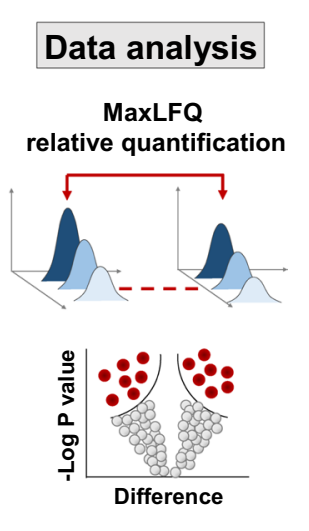

Marker candidate proteins Not altered proteins b

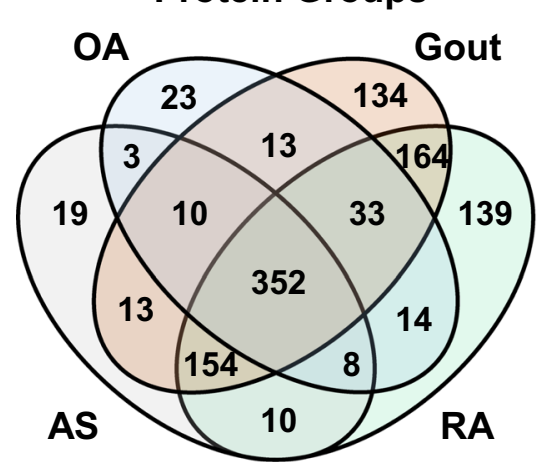

Peptides

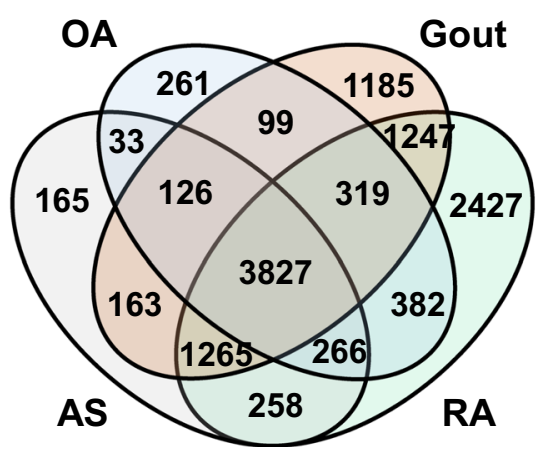

Fig. 1 a Schematic diagram of proteomics experimental workflow. Synovial fluids of ankylosing spondylitis (AS), osteoarthritis (OA), gout, and rheumatoid arthritis (RA) were subject to immunodepletion using the multiple affinity removal system (MARS) and followed by trypsinization and LC-MS/MS analysis. MaxQuant LFQ (MaxLFQ) analysis was carried out for quantitative comparison. b Venn diagram showing the unique and shared protein groups and peptides identified in the synovial fluid of each group

(obtained in June 2018). Carbamidomethylation of cysteine was selected as a fixed modification, while $\mathrm{N}$-terminal acetylation and methionine oxidation were set as variable modifications. $1 \%$ of false discovery rate (FDR) cutoff was applied at the levels of peptide-spectrum match and protein. An initial precursor mass deviation of up to $4.5 \mathrm{ppm}$ and a fragment mass deviation of up to $20 \mathrm{ppm}$ were allowed. To maximize the protein identification in initial discovery stage, the criterion with minimum stringency, at least one unique peptide, was applied for the protein identification. Proteins were quantified using the label-free quantification (LFQ) algorithm in MaxQuant [22]. The option of 'match between runs' was used for nonlinear retention time alignment. The match time window was $0.7 \mathrm{~min}$, and the alignment time window was $20 \mathrm{~min}$. Further statistical and bioinformatics analysis was performed using Perseus software (v.1.5.3.2) [23]. Proteins hit to the decoy database and contaminants, or proteins only identified by the peptide with modification sites such as methionine oxidation were filtered out prior to further analysis. A minimum of three valid protein quantification values across each clinical group was required for protein quantification. The LFQ intensity of individual proteins in the AS group was compared with that of such proteins in the other clinical groups following log2 transformation, i.e., AS vs OA, AS vs gout, and AS vs RA. Statistical analysis of the log2-transformed data was performed using two samples t-test ( $p$ value $<0.05$ ). Proteins with fold change $\geq 1.5$ or $\leq 0.67$ between AS and other patient groups were considered to be differentially expressed proteins (DEPs) for AS group. The resulting DEPs were submitted to gene ontology (GO) annotation enrichment analysis. 


\section{Enrichment and network analysis}

To explore functional enrichment in the identified proteins in each of the clinical groups, GO analysis was performed using DAVID (version 6.8) [24]. We identified biological processes and KEGG pathways [25] that were enriched in our DEP list with p values of less than 0.05 . To construct a network representing the enriched GO terms, we selected DEPs that are involved in the enriched cellular processes. We then built a protein network model using protein interaction information obtained from STRING version 11 database [26]. The interaction network models were visualized using Cytoscape [27].

\section{Western blot verification}

We carried out western blot based verification experiments for the candidates as AS-specific proteins from LC-MS/MS proteomic data. To reduce the viscosity of SF, samples were treated with hyaluronidase (Sigma, H3884) at room temperature for 5 or $10 \mathrm{~min}$, and then diluted 1:10 in RIPA buffer (Thermo Fisher) containing both protease and phosphatase inhibitor (Roche Diagnostics $\mathrm{GmbH}$ ). The diluted sample was incubated on ice for five $\mathrm{min}$, then transferred into new tube and centrifuged at $10,000 \times g$ at $4{ }^{\circ} \mathrm{C}$ for $15 \mathrm{~min}$. Equal amounts of protein $(30 \mu \mathrm{g})$ in each sample were aliquoted following protein quantitation by BCA assay (Thermo Fisher). Samples were mixed with sodium dodecyl sulfate (SDS) loading buffer and separated using SDS polyacrylamide gel electrophoresis (Western Blotting Kit, Hoefer Inc.). Proteins were then transferred onto nitrocellulose membranes (Amersham), blocked with 5\% non-fat dried milk in tris-buffered saline containing $0.05 \%$ Tween-20 and immunoblotted with the appropriate primary and secondary antibodies. The antibodies used were as follows: rabbit polyclonal antibodies for C9 (1:5000, PA5-29093, Thermo Fisher), CFHR5 (1:500, ab175254, Abcam), MMP3 (1:5000, ab52915, Abcam), mannose-binding protein C (MBL2) (1:5000, ab189856, Abcam), complement C4-A (C4A) (1:5000, ab66790, Abcam), serum amyloid P-component (APCS) (1:5000, ab45151, Abcam), matrix metalloproteinase-1 (MMP1) (1:3000, ab38929, Abcam), anti-transferrin (1:5000, ab109503, Abcam), and peroxidase-conjugated AffiniPure Donkey anti-rabbit IgG $(\mathrm{H}+\mathrm{L})(1: 10,000$, Jackson Immunoresearch). Anti-transferrin $(77 \mathrm{kDa})$ was used as a loading control [28].

\section{Results}

\section{General characteristics of study subjects}

The summary statistics and general characteristics of the study subjects are presented in Table 1 and Additional file 1: Table S1, respectively. The AS group was 32.6 $( \pm 10.6)$ years of age, with $7.0( \pm 4.6)$ years mean disease duration, 80\% HLA-B27 positive rate, 20\% biologics utilization, $59.5( \pm 39.7) \mathrm{mm} / \mathrm{h}$ averaged ESR, and $4.9( \pm 4.3)$ $\mathrm{mg} / \mathrm{dL}$ averaged CRP. The RA group were all females at $59.6( \pm 11.6)$ years of age, with $4.9( \pm 4.9)$ years mean disease duration, $90 \%$ rheumatoid factor and anti-CCP antibody positive rate, $30 \%$ biologics utilization, 32.7 ( \pm 17.0$)$ $\mathrm{mm} / \mathrm{h}$ averaged ESR, and $0.5( \pm 3.7) \mathrm{mg} / \mathrm{dL}$ averaged CRP. The gout group were all males at $62.8( \pm 12.8)$ years of age, with $0.3( \pm 0.9)$ years of disease duration, and 7.1 $( \pm 2.6) \mathrm{mg} / \mathrm{dL}$ concentration of serum uric acid. The OA group were $64.8( \pm 8.5)$ years of age, with $5.5( \pm 5.9)$ years mean disease duration.

\section{Proteomic analysis of SF from AS, RA, gout, and OA patient groups}

To obtain insight into the molecular basis of AS in the SF, we performed a LFQ intensity-based proteomic comparison of SF from AS patients and SF from patients with OA, gout, and RA (Fig. 1a). All SFs were first subjected to immunodepletion of the 14 most abundant proteins using an MARS kit, and then followed by LC-MS/MS experiments, resulting in the identification of 569 proteins and 6103 peptides in the AS group, 874 proteins and 9991 peptides in the RA group, 456 proteins and 5313 peptides in the OA group, and 873 proteins and 8231 peptides in the gout group at $<1 \%$ FDR (Fig. 1b). A further MaxQuant analysis using 'match between runs' to minimize missing quantities between replicates and groups $[21,29]$ was then carried out. To resolve the ASspecific DEPs against other disease groups, we performed a series of pairwise statistical comparisons of AS group versus three different control groups (OA, gout and RA) based on the LFQ intensity of commonly identified proteins from $\geq 3$ patients in each group using the Perseus software, resulting in 385,500 , and 485 quantifiable proteins for AS vs OA, AS vs gout, and AS vs RA comparison sets, respectively (Additional file 2: Table S2). DEPs in such three comparison sets were defined as the proteins with $>1.5$ fold-change and $<0.05 p$ value and the corresponding numbers of up- and down-regulated DEPs were 102 and 84 for AS/OA set, 41 and 179 for AS/gout set, and 69 and 151 for AS/RA set, respectively (Additional file 3: Table S3). Intriguingly, we found that eight of the DEPs were AS-specifically up-regulated (HP, MMP1, MMP3, APCS, CFHR5, C9, C4A, and MBL2 in Table 2), whereas a total of 24 proteins including immune-related proteins such as PLA2G2A (Phospholipase A2) were commonly downregulated in AS samples against three other arthritides (Fig. 2a).

Gene ontology analysis to clarify the molecular basis for AS Next, we tried to gain insight into the functional roles of the DEPs associated with AS (Fig. 2a and Table 2) via GO 


\begin{tabular}{|c|c|c|c|c|}
\hline Gene symbol & Protein & $\begin{array}{l}\text { Fold change } \\
\text { (AS/RA) }\end{array}$ & $\begin{array}{l}\text { Fold change } \\
\text { (AS/Gout) }\end{array}$ & $\begin{array}{l}\text { Fold change } \\
\text { (AS/OA) }\end{array}$ \\
\hline HP & Haptoglobin & 2.67 & 5.93 & 27.97 \\
\hline MMP3 & Matrix metalloproteinase-3 & 2.02 & 2.63 & 10.13 \\
\hline CFHR5 & Complement factor H-related protein 5 & 1.61 & 4.52 & 3.23 \\
\hline C9 & Complement component C9 & 1.85 & 1.75 & 1.82 \\
\hline MBL2 & Mannose-binding protein C & 1.68 & 1.52 & 1.61 \\
\hline C4A & Complement C4-A & 1.92 & 2.02 & 1.63 \\
\hline APCS & Serum amyloid P-component & 15.69 & 4.81 & 6.53 \\
\hline MMP1 & Matrix metalloproteinase-1 & 1.97 & 2.75 & 14.96 \\
\hline
\end{tabular}

AS: ankylosing spondylitis; OA: osteoarthritis; RA: rheumatoid arthritis

analysis of the up- and downregulated DEPs (Additional file 4: Table S4). Interestingly, the AS-specifically upregulated proteins showed enriched patterns largely to the immune response-related biological processes (Fig. 2b). In detail, the AS-specifically upregulated proteins vs OA or gout were strongly associated with the innate immune response (Fig. 2b). The enriched GO terms suggested that the AS-specifically upregulated proteins may play an important role in the complement activation and the inflammatory response. In addition, the GO terms involved in metabolic processes, protein oligomerization, proteolysis, and extracellular matrix disassembly were also enriched in the AS-specific DEPs. On the contrary, the GO terms related to various biological processes including extracellular matrix organization, collagen fibril organization, and cellular structure were enriched in the AS-specifically downregulated DEPs (Fig. 2c).

\section{Protein-protein interaction network describing AS}

To better understand the cellular networks that are altered in the synovial fluid of AS patients, we created protein-protein interaction network models of ASspecific upregulated DEPs using the STRING database (Fig. 3). Four important processes were enriched in the AS-specific upregulated DEPs: innate immune response, complement activation, platelet degranulation, and glycolytic process. We found that APCS (innate immune response), C9, C4A, CFHR5, and MBL2 (complement activation), which are common in AS SF, are known to interact based on this network.

\section{Verification by western blot analysis}

Following the LC-MS/MS analysis, the levels of 7 DEPs in SF (MMP3 $(50 \mathrm{kDa})$, CFHR5 $(70 \mathrm{kDa}), \mathrm{C} 9(60 \mathrm{kDa})$, MBL2 (26 kDa), C4A (95 kDa), APCS (25 kDa), and MMP1 (50 kDa), except for HP, which occupies a large portion in synovial fluid) were verified by western blot using specific primary antibodies. Figure 4 clearly showed that the three candidates (MMP3, CFHR5, and C9) are reproducibly represented as AS-specific proteins in both original (Fig. 4a) and additional independent (Fig. 4b) sample sets, further verifying the results from mass spectrometry. MMP3 was considered as a positive control because it has been known to be highly expressed in the SF of AS patients [30], expectedly showing the significantly increased expression levels in AS SF compared to those in the other clinical groups. MMP1 protein was excluded due to the vain western blot detection and transferrin protein was included in western blot experiments as a control of loading amount.

However, the AS-specifically upregulated expressions of the remaining three proteins (C4A, MBL2 and APCS) in mass spectrometry were not clearly verified by the original western blot experiments (Additional file 5: Figure 1a). Intriguingly, C4A protein showed a clear AS-specific pattern in the independent sample set (Additional file 5: Figure $1 \mathrm{~b}$ ), suggesting that the $\mathrm{C} 4 \mathrm{~A}$ protein needs to be further verified or included to the validation candidates of AS-specific markers.

\section{Discussion}

Early diagnosis of AS is difficult because the etiology is not clear and there is no specific diagnostic indicator [31]. Mass spectrometry-based proteomics is a state-ofthe-art analytical technique that enables the discovery of indicator proteins for the diagnosis and treatment of diseases. There has been a recent expansion in proteomics research on a number of different rheumatic diseases [32].

In the present study, we performed comprehensive proteomic profiling and western blot analysis of SF obtained from patients with AS and SF obtained from patients with comparative diseases (RA, gout, and OA). SF samples obtained from patients with AS and the three 
a
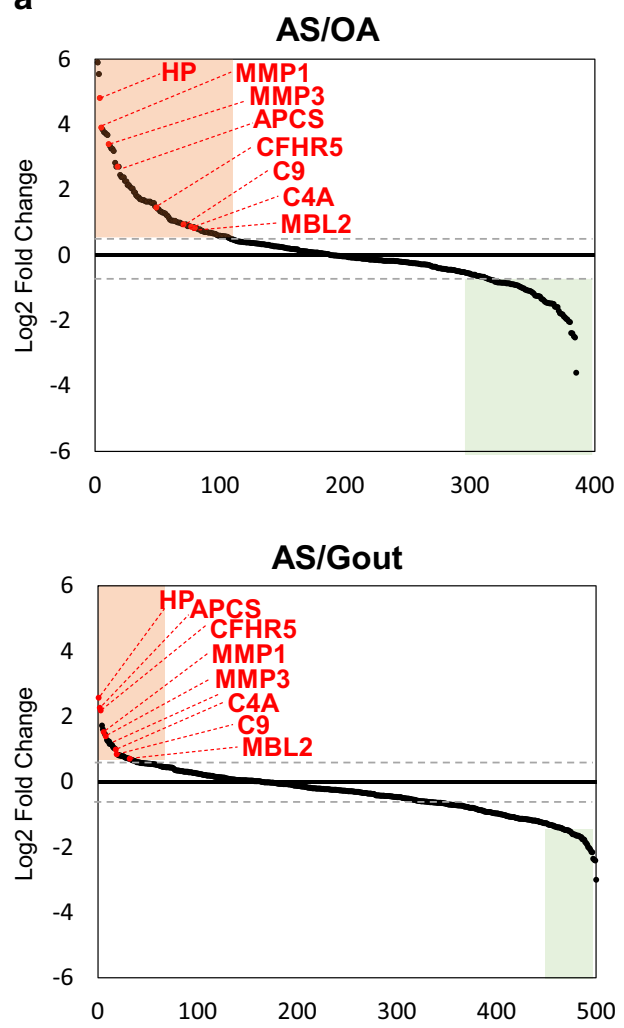

AS/RA

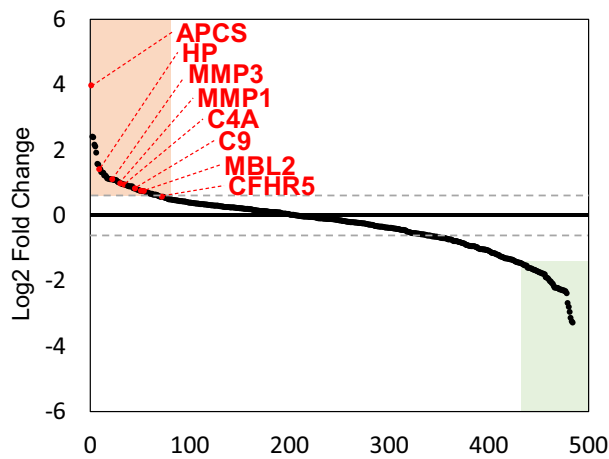

b

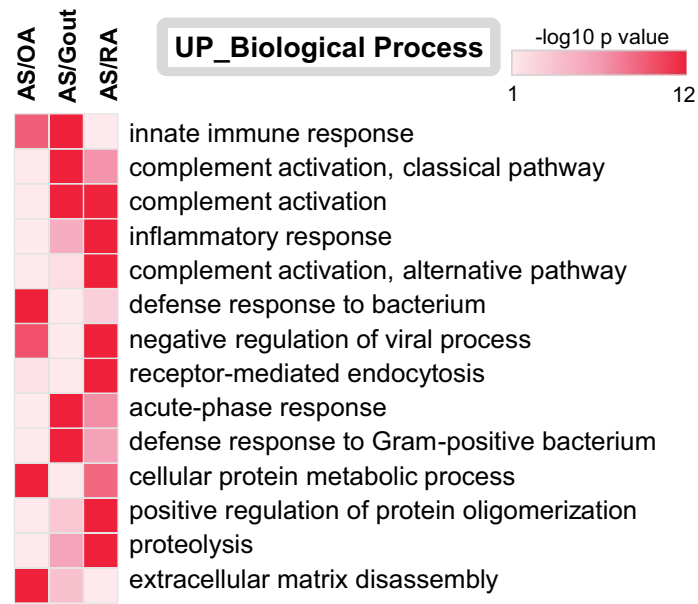

C

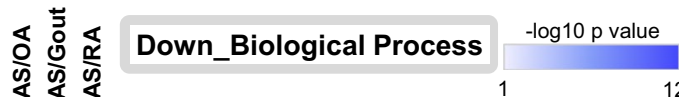

extracellular matrix organization extracellular matrix disassembly platelet degranulation collagen fibril organization collagen catabolic process positive regulation of stress fiber assembly angiogenesis

leukocyte migration blood vessel endothelial cell migration lipid transport retinoid metabolic process retina homeostasis response to drug response to estradiol response to ethanol response to peptide hormone cellular response to amino acid stimulus cellular protein metabolic process integrin-mediated signaling pathway negative regulation of receptor binding negative regulation of endopeptidase activity osteoblast differentiation peripheral nervous system axon regeneration

Fig. 2 a Reverse cumulative plots of protein fold change in the synovial fluid from each disease group. The most commonly upregulated 8 proteins (HP, MMP1, MMP3, APCS, CFHR5, C9, C4A, and MBL2) are highlighted. b, c Gene ontology (GO) analysis of differentially expressed proteins in the synovial fluid of the AS group compared to in the OA, gout, and RA groups. Heatmaps showing significantly enriched GO biological process terms $(p<0.05)$ for differentially expressed proteins. The red color in the heatmap indicates a significant upregulation (b) and blue color indicates downregulation of the biological process (c)

disease control groups were quantified using highly sensitive LC-MS/MS and LFQ-based analysis. We discovered eight biomarker candidate proteins (HP, MMP3, CFHR5, C9, MBL2, C4A, APCS, and MMP1) with > 1.5 of fold change in AS compared to that in the other groups. Western blot experiments for seven proteins (with the exception of HP) were performed to further verify the differential expression level. As a result, three proteins (MMP3, CFHR5 and C9) were solidly verified to be highly expressed in the SF of patients with AS.

MMP family proteins are involved in the pathogenesis of arthritis. In particular, MMP3 is a protease that is synthesized and secreted by fibroblasts and chondrocytes in synovial joints, and also activates other MMPs 


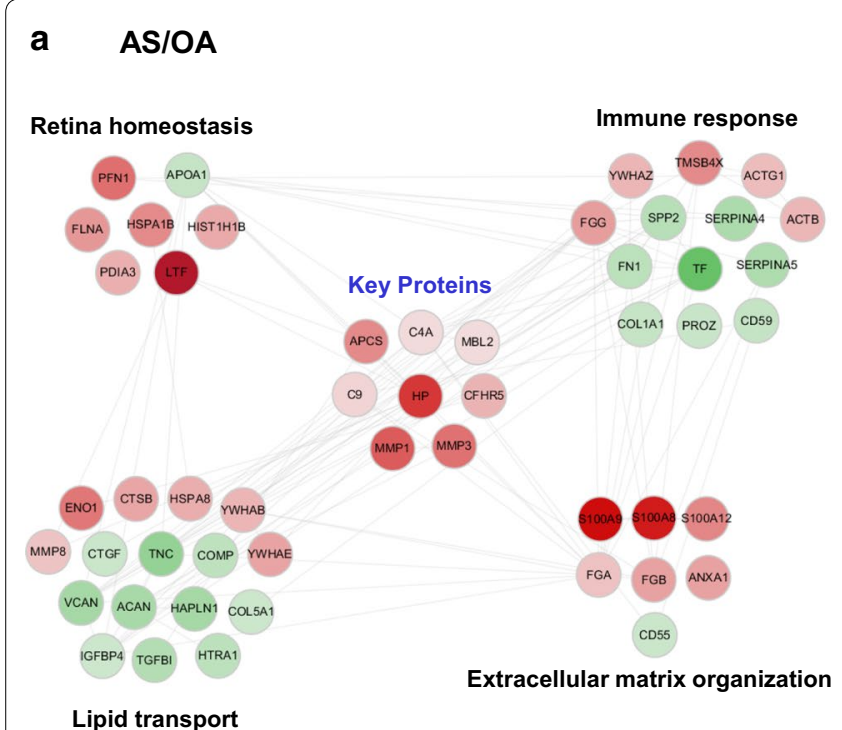

b AS/Gout
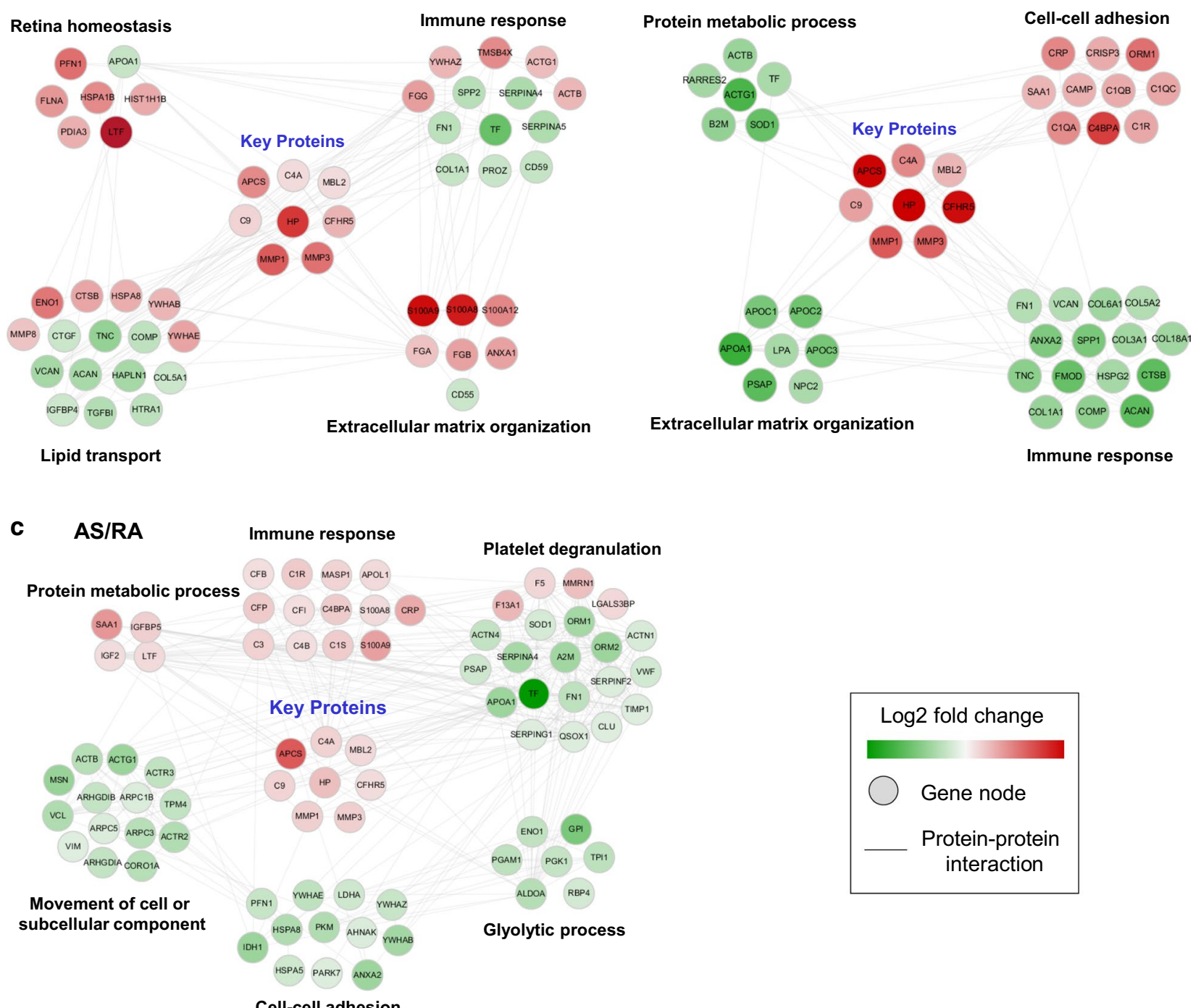

Fig. 3 Network modelling of differentially expressed proteins in the synovial fluid of the AS group versus the $\mathbf{a} O A, \mathbf{b}$ gout and $\mathbf{c}$ RA group. Proteinprotein interaction network showing the biological processes affected, including the immune response, platelet degranulation, cell-cell adhesion etc. The colors of the nodes represent proteins with increased (red) or decreased (green) levels in the synovial fluid of the AS group compared to in the other groups. The connection between nodes (solid grey lines) shows either a regulatory role or physical interaction between proteins

such as MMP1, MMP7, and MMP9 [33]. A recent metaanalysis suggests that the serum levels of MMP3 rise in AS patients [29]. The validity of our results was further enhanced by the discovery of previously researched AS relevant protein, MMP3.

CFHR5 plays an important role in the alternative pathway complement system, and binds to $\mathrm{C} 3$ to activate it [34]. This protein is highly expressed in the serum of AS patients [35]. In addition, high concentrations of CFHR5 were observed in the plasma of patients with systemic lupus erythematosus, an autoimmune disease [36]. C9 is a member of the membrane attack complex (MAC) complement system, and causes lysis by inducing pores in the cell membrane after activating $\mathrm{C} 5$, the final stage of the complement system [37]. The levels of this protein are elevated in the serum of AS patients [35].

Unlike the proteomic result, MBL2 showed inconsistently and slightly upregulated expression level according to the western blotting results. The expression of MBL2 was observed in 11 out of 15 cases of AS in the western blot, compared to six in RA, six in gout, and three in degenerative arthritis. MBL2 has an ability to 


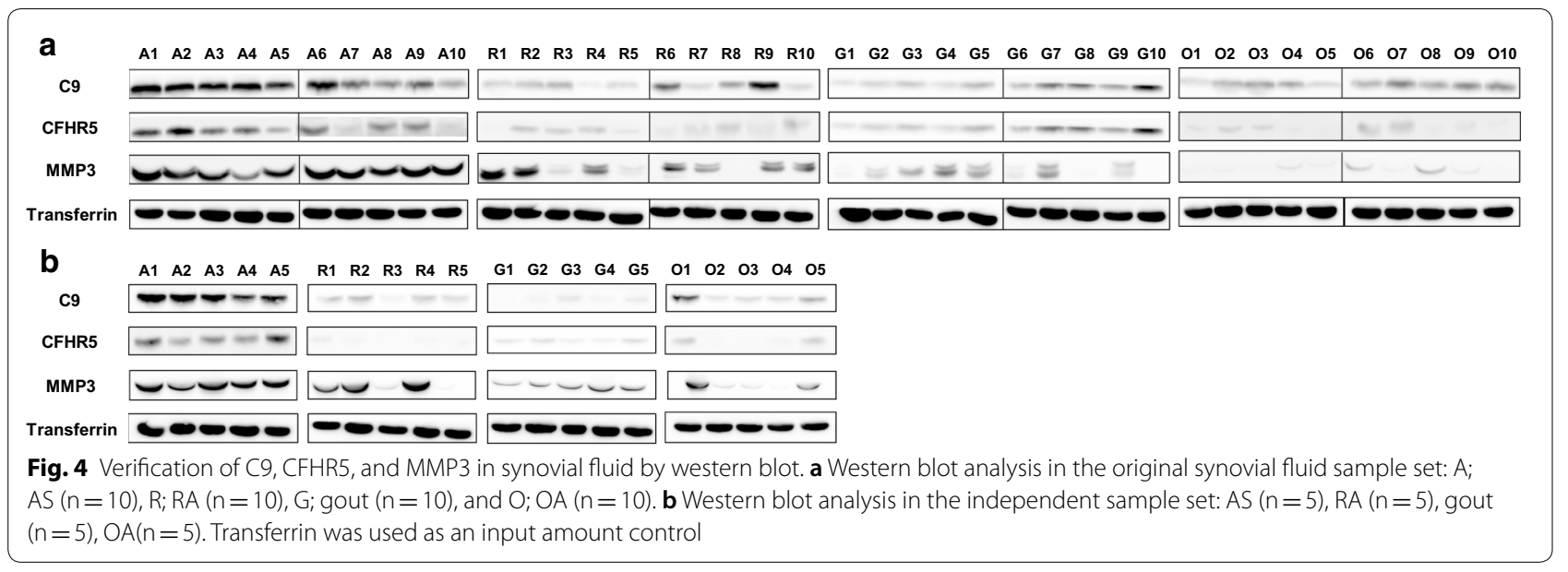

recognize carbohydrate types found on pathogenic microbial surfaces. MBL2 initiates the lectin pathway and opsonizes apoptotic and necrotic cells [38].

C4A, a complementary protein with MBL2, showed vague expression pattern in the original western blot set, while a clear AS-specific pattern in the independent western blot set. C4A is involved in the classical pathway of the complement system. Deficiency of $\mathrm{C} 4 \mathrm{~A}$ is associated with systemic lupus erythematosus and type 1 diabetes mellitus, and its overexpression is associated with mental disorders such as schizophrenia and bipolar disorder [39]. The complement system is an important mechanism of humoral and innate immunity. Suppressing the complement system in an animal model of AS may improve AS treatment [40]. The complement system is active in patients with systemic sclerosis, a musculoskeletal disorder [41].

APCS is associated with the innate immune system and is known to have increased expression in the SF and serum of AS patients [35, 42]. However, APCS showed quite consistent expression patterns for all SF samples in western blot data, even though MS-based proteomics revealed significantly higher fold-changes of APCS protein in the AS group than the other groups (AS/RA: 15.69, AS/gout: 4.81, AS/OA: 6.53) (Table 2). Previously, CRP and APCS were shown to have 51\% sequence homology in rats [43]. In this regard, the antibody used in this study might not be exclusively specific to APCS, simultaneously detecting other inflammatory markers too. The antibody-based verification approach may therefore not be suitable in all cases, and an alternative approach may be better for this particular protein. For instance, targeted proteomics such as multiple reaction monitoring can be an alternative. The last candidate, MMP1, a protein mainly expressed in bone metabolism, was not detected by western blot in all disease groups, and should be verified further with other approach.

The limitations of the present study are the following: the relatively small number of patients included due to the difficulty in obtaining clinical sample donor and the lack of further validation method beyond western blotting. Further validation will increase the likelihood of identifying biomarkers for AS, as will increasing the number of samples and using alternative sample types including urine, serum, synovial membrane, and animal models.

\section{Conclusions}

In total, 1089 proteins were identified by label-free comparative proteomic analysis in the SF of patients with AS, RA, gout, and OA. This is the largest dataset of proteins identified in the synovial fluid to date. This is also the first time that three diseases (RA, gout, and OA) have been used simultaneously as disease control groups during proteomic profiling of AS. Out of the significantly dysregulated proteins, eight were significantly increased in the SF of AS patients compared to that of patients of the other three diseases. Out of these, MBL2 and $\mathrm{C} 4 \mathrm{~A}$ were the first proteins previously reported as AS markers, while the remaining six proteins were first reported as AS markers in SF sample in this study. Four out of the eight proteins are part of the complement system, which appears to be highly associated with AS. Altogether, these results suggest an important role for complement signaling during AS disease progression, and this avenue of enquiry may provide insight into the underlying molecular mechanisms of AS. The clinical utility of the putative biomarkers identified in the present study should be further validated in a larger cohort. 


\section{Supplementary information}

Supplementary information accompanies this paper at https://doi. org/10.1186/s12014-020-09281-y.

Additional file 1: Table S1. Baseline characteristics of the first patient cohort used for the LC-MS/MS experiments and the associated western blot verification (a), and the second patient cohort used for the western blot verification (b).

Additional file 2: Table S2. Quantifiable protein list of AS patient group versus three different control groups (OA, gout and RA), showing 385, 500, and 485 quantifiable proteins for AS vs OA, AS vs gout, and AS vs RA comparison sets, respectively. Protein accession number, gene symbols, fold-change, and $p$-value are provided.

Additional file 3: Table S3. Differentially expressed proteins (DEPS) in AS vs OA, AS vs gout, and AS vs RA comparison sets, defined as the proteins with $>1.5$-fold-change and $<0.05 p$-value. Gene symbols, Log2 foldchange and descriptions of the proteins are provided.

Additional file 4: Table S4. Gene ontology analysis in terms of biological process performed by DAVID tool for the up-regulated $(\mathrm{a}-\mathrm{c})$ or and down-regulated DEPs (d-f) in OA, Gout, and RA groups, versus AS group, respectively.

Additional file 5: Figure S1. Verification of C4A, MBL2, and APCS in synovial fluid by western blot. (a) Western blot analysis in the original synovial fluid sample set: A; AS ( $n=10), R$; RA $(n=10), G$; gout $(n=10)$, and O; OA $(n=10)$. (b) Western blot analysis in the Independent sample set: AS $(n=5), R A(n=5)$, gout $(n=5), O A(n=5)$. Transferrin was used as an input amount control.

\section{Abbreviations}

AS: Ankylosing spondylitis; SF: Synovial fluid; OA: Osteoarthritis; RA: Rheumatoid arthritis; HLA: Human leukocyte antigen; TNF: Tumor necrosis factor; CCP: Cyclic citrullinated peptide; AGC: Automated gain control; FDR: False discovery rate; LFQ: Label-free quantification; DEP: Differentially expressed proteins; SDS: Sodium dodecyl sulfate; bDMARD: Biologic disease-modifying anti-rheumatic drug; CDMARD: Conventional disease-modifying anti-rheumatic drug; CRP: C-reactive protein; ETN: Etanercept; ESR: Erythrocyte sedimentation rate; HP: Haptoglobin; MMP1: Matrix metalloproteinase-1; MMP3: Matrix metalloproteinase-1; APCS: Serum amyloid P-component; CFHR5: Complement factor H-related protein 5; C9: Complement component C9; C4A: Complement C4-A; MBL2: Mannose-binding protein C; MAC: Membrane attack complex.

\section{Acknowledgements}

The biospecimens used in the present study were provided by the Keimyung University Dongsan Hospital Korea Regional Biobank, a member of the National Biobank of Korea, which is supported by the Ministry of Health and Welfare.

\section{Authors' contributions}

CNS and JSK conceived and designed the study. JHL and JHJ performed experiments with a support of JK and JR. JHJ, JK, KPK and JSK performed proteomics data analysis. JHL, SHK, WKB, THK, and CNS clinically interpreted the results. CNS, JSK, KPK, JHL, JHJ, and JSR wrote the manuscript. All authors read and approved the final manuscript.

\section{Funding}

This work was supported by NFR-2017R1C1B5017278 (CNS) and NRF2018M3C1B7020722 (SHK) of the National Research Foundation, and IBSR008-D1 (JSK) of Institute for Basic Science from the Ministry of Science and ICT of Korea.

\section{Availability of data and materials}

All of the raw data has been deposited into the PRIDE Archive (ProteomeXchange) with the data set identifier PXD016620.

\section{Ethics approval and consent to participate}

The study was approved by the ethical committee of the Keimyung University Dongsan Hospital (IRB 2015-12-022).

\section{Consent for publication}

Not applicable.

\section{Competing interests}

The authors declare that they have no competing interests.

\section{Author details}

${ }^{1}$ Division of Rheumatology, Department of Internal Medicine, School of Medicine, Keimyung University, Daegu, South Korea. ${ }^{2}$ Department of Applied Chemistry, Institute of Natural Science, Global Center for Pharmaceutical Ingredient Materials, Kyung Hee University, Yongin, South Korea. ${ }^{3}$ Center for RNA Research, Institute of Basic Science (IBS), Seoul 08826, South Korea. ${ }^{4}$ School of Biological Sciences, Seoul National University, Seoul 08826, South Korea. ${ }^{5}$ Department of Microbiology, School of Medicine, Keimyung University, Daegu, South Korea. ${ }^{6}$ New Drug R\&D Center, ARIBIO Co. Ltd., Seongnam, South Korea. ${ }^{7}$ Department of Rheumatology, Hanyang University Hospital for Rheumatic Diseases, Seoul, South Korea.

Received: 23 December 2019 Accepted: 15 May 2020

Published online: 01 June 2020

\section{References}

1. Raychaudhuri SP, Deodhar A. The classification and diagnostic criteria of ankylosing spondylitis. J Autoimmun. 2014;48-49:128-33.

2. Ranganathan V, Gracey E, Brown MA, Inman RD, Haroon N. Pathogenesis of ankylosing spondylitis-recent advances and future directions. Nat Rev Rheumatol. 2017;13(6):359-67.

3. Zink A, Braun J, Listing J, Wollenhaupt J, Ctr GCA. Disability and handicap in rheumatoid arthritis and ankylosing spondylitis - results from the German rheumatological database. J Rheumatol. 2000;27(3):613-22.

4. Boonen $\mathrm{A}$, Chorus $\mathrm{A}$, Miedema $\mathrm{H}$, van der Heijde $\mathrm{D}$, van der Tempel $\mathrm{H}$, van der Linden S. Employment, work disability, and work days lost in patients with ankylosing spondylitis: a cross sectional study of Dutch patients. Ann Rheum Dis. 2001;60(4):353-8.

5. Sieper J, Braun J, Rudwaleit M, Boonen A, Zink A. Ankylosing spondylitis: an overview. Ann Rheum Dis. 2002;61:8-18.

6. Gladman DD. Clinical aspects of the spondyloarthropathies. Am J Med Sci. 1998;316(4):234-8.

7. Smith MD. The normal synovium. Open Rheumatol J. 2011;5:100-6.

8. Singh N, Vogelgesang SA. Monoarticular Arthritis. Med Clin N Am. 2017;101(3):607-13.

9. Gobezie R, Kho A, Krastins B, Sarracino DA, Thornhill TS, Chase M, et al. High abundance synovial fluid proteome: distinct profiles in health and osteoarthritis. Arthritis Res Ther. 2007;9(2):R36.

10. Mahendran SM, Oikonomopoulou K, Diamandis EP, Chandran V. Synovial fluid proteomics in the pursuit of arthritis mediators: an evolving field of novel biomarker discovery. Crit Rev Cl Lab Sci. 2017;54(7-8):495-505.

11. Park YJ, Chung MK, Hwang D, Kim WU. Proteomics in Rheumatoid Arthritis Research. Immune Netw. 2015;15(4):177-85.

12. Mohan C, Assassi S. Biomarkers in rheumatic diseases: how can they facilitate diagnosis and assessment of disease activity? Bmj-Brit Med J. 2015;351:h5079.

13. Liao WX, Li ZL, Li TS, Zhang Q, Zhang H, Wang XZ. Proteomic analysis of synovial fluid in osteoarthritis using SWATH-mass spectrometry. Mol Med Rep. 2018;17(2):2827-36.

14. Peffers MJ, Smagul A, Anderson JR. Proteomic analysis of synovial fluid: current and potential uses to improve clinical outcomes. Expert Rev Proteomic. 2019;16(4):287-302.

15. Castro-Santos P, Laborde CM, Diaz-Pena R. Genomics, proteomics and metabolomics: their emerging roles in the discovery and validation of rheumatoid arthritis biomarkers. Clin Exp Rheumatol. 2015;33(2):279-86.

16. Arnett FC, Edworthy SM, Bloch DA, Mcshane DJ, Fries JF, Cooper NS, et al. The American-Rheumatism-Association 1987 Revised Criteria for the Classification of Rheumatoid-Arthritis. Arthritis Rheum. 1988;31(3):315-24.

17. Vanderlinden S, Valkenburg HA, Cats A. Evaluation of diagnostic-criteria for ankylosing-spondylitis - a proposal for modification of the New-York Criteria. Arthritis Rheum. 1984;27(4):361-8.

18. Altman R, Asch E, Bloch D, Bole G, Borenstein D, Brandt $K$, et al. Development of criteria for the classification and reporting of 
osteoarthritis — classification of osteoarthritis of the Knee. Arthritis Rheum. 1986;29(8):1039-49.

19. Wallace SL, Robinson H, Masi AT, Decker JL, McCarty DJ, Yu TF. Preliminary criteria for the classification of the acute arthritis of primary gout. Arthritis Rheum. 1977;20(3):895-900.

20. Geyer PE, Voytik E, Treit PV, Doll S, Kleinhempel A, Niu LL, et al. Plasma Proteome Profiling to detect and avoid sample-related biases in biomarker studies. Embo Mol Med. 2019;11(11):e10427.

21. Cox J, Neuhauser N, Michalski A, Scheltema RA, Olsen JV, Mann M. Andromeda: a peptide search engine integrated into the MaxQuant environment. J Proteome Res. 2011;10(4):1794-805.

22. Cox J, Hein MY, Luber CA, Paron I, Nagaraj N, Mann M. Accurate Proteome-wide Label-free Quantification by Delayed Normalization and Maximal Peptide Ratio Extraction. Termed MaxLFQ. Mol Cell Proteomics. 2014;13(9):2513-26.

23. Tyanova S, Temu T, Sinitcyn P, Carlson A, Hein MY, Geiger T, et al. The Perseus computational platform for comprehensive analysis of (prote)omics data. Nat Methods. 2016;13(9):731-40.

24. Huang DW, Sherman BT, Lempicki RA. Systematic and integrative analysis of large gene lists using DAVID bioinformatics resources. Nat Protoc. 2009;4(1):44-57.

25. Kanehisa M, Furumichi M, Tanabe M, Sato Y, Morishima K. KEGG: new perspectives on genomes, pathways, diseases and drugs. Nucleic Acids Res. 2017;45(D1):D353-61.

26. Franceschini A, Szklarczyk D, Frankild S, Kuhn M, Simonovic M, Roth A, et al. STRING v9.1: protein-protein interaction networks, with increased coverage and integration. Nucleic Acids Res. 2013;41(D1):D808-15.

27. Shannon P, Markiel A, Ozier O, Baliga NS, Wang JT, Ramage D, et al. Cytoscape: a software environment for integrated models of biomolecular interaction networks. Genome Res. 2003;13(11):2498-504.

28. Minagawa I, Fukuda M, Ishige H, Kohriki H, Shibata M, Park EY, et al. Relaxin-like factor (RLF)/insulin-like peptide 3 (INSL3) is secreted from testicular Leydig cells as a monomeric protein comprising three domains B-C-A with full biological activity in boars. Biochem J. 2012;441:265-73.

29. Cox J, Mann M. MaxQuant enables high peptide identification rates, individualized p.p.b.-range mass accuracies and proteome-wide protein quantification. Nat Biotechnol. 2008;26(12):1367-72.

30. Gao JW, Zhang KF, Lu JS, Su T. Serum matrix metalloproteinases-3 levels in patients with ankylosing spondylitis. Genet Mol Res. 2015;14(4):17068-78.

31. Zochling J, Braun J. Quality indicators, guidelines and outcome measures in ankylosing spondylitis. Clin Exp Rheumatol. 2007;25(6):S147-52.

32. Cai AJ, Qi SW, Su ZW, Shen HQ, Yang Y, He L, et al. Quantitative proteomic analysis of peripheral blood mononuclear cells in ankylosing spondylitis by iTRAQ. Cts-Clin Transl Sci. 2015;8(5):579-83.
33. Lerner A, Neidhofer $S$, Reuter $S$, Matthias T. MMP3 is a reliable marker for disease activity, radiological monitoring, disease outcome predictability, and therapeutic response in rheumatoid arthritis. Best Pract Res $\mathrm{Cl}$ Rh. 2018;32(4):550-62.

34. Skerka C, Chen Q, Fremeaux-Bacchi V, Roumenina LT. Complement factor $\mathrm{H}$ related proteins (CFHRs). Mol Immunol. 2013;56(3):170-80.

35. Fischer R, Trudgian DC, Wright C, Thomas G, Bradbury LA, Brown MA, et al. Discovery of candidate serum proteomic and metabolomic biomarkers in ankylosing spondylitis. Mol Cell Proteomics. 2012;11(2):M111.013904.

36. Hu XF, Liu HD, Du J, Chen Y, Yang M, Xie YH, et al. The clinical significance of plasma CFHR 1-5 in lupus nephropathy. Immunobiology. 2019:224(3):339-46.

37. Franc $V$, Yang Y, Heck AJR. Proteoform profile mapping of the human serum complement component C9 revealing unexpected new features of N-, O-, and C-Glycosylation. Anal Chem. 2017;89(6):3483-91.

38. Skare TL, Nisihara R, Cieslinski JZ, Zeni JO, Rasera HN, Messias-Reason I, et al. Mannose-binding lectin deficiency in Brazilian patients with spondyloarthritis. Immunol Invest. 2017;46(2):183-9.

39. Ingram G, Hakobyan S, Hirst CL, Harris CL, Loveless S, Mitchell JP, et al. Systemic complement profiling in multiple sclerosis as a biomarker of disease state. Mult Scler J. 2012;18(10):1401-11.

40. Yang CQ, Ding PP, Wang QK, Zhang L, Zhang X, Zhao JQ, et al. Inhibition of complement retards ankylosing spondylitis progression. Sci Rep-Uk. 2016:6:34643.

41. Okroj M, Johansson M, Saxne T, Blom AM, Hesselstrand R. Analysis of complement biomarkers in systemic sclerosis indicates a distinct pattern in scleroderma renal crisis. Arthritis Res Ther. 2016:18:267.

42. Bhattacharjee M, Sharma R, Goel R, Balakrishnan L, Renuse S, Advani $J$, et al. A multilectin affinity approach for comparative glycoprotein profiling of rheumatoid arthritis and spondyloarthropathy. Clin Proteom. 2013;10:11.

43. Floydsmith $G$, Whitehead AS, Colten HR, Francke U. The human C-reactive protein gene (Crp) and serum amyloid-P component gene (Apcs) are located on the proximal long arm of chromosome-1. Immunogenetics. 1986;24(3):171-6.

\section{Publisher's Note}

Springer Nature remains neutral with regard to jurisdictional claims in published maps and institutional affiliations.
Ready to submit your research? Choose BMC and benefit from:

- fast, convenient online submission

- thorough peer review by experienced researchers in your field

- rapid publication on acceptance

- support for research data, including large and complex data types

- gold Open Access which fosters wider collaboration and increased citations

- maximum visibility for your research: over $100 \mathrm{M}$ website views per year

At BMC, research is always in progress.

Learn more biomedcentral.com/submissions 